\title{
Aberrant promoter methylation profiles and association with survival in patients with hepatocellular carcinoma
}

This article was published in the following Dove Press journal:

OncoTargets and Therapy

8 May 2017

Number of times this article has been viewed

\author{
Dani Zhong \\ Hong Cen \\ Department of Chemotherapy, \\ Tumor Hospital of Guangxi Medical \\ University, Nanning, Guangxi, People's \\ Republic of China
}

\begin{abstract}
The aim of this study was to investigate the prognostic and diagnostic value of genes with promoter methylation in hepatocellular carcinoma (HCC) patients. On the basis of The Cancer Genome Atlas data, we identified genes with differentially methylated promoters in HCC tissues and adjacent non-tumor tissues, using the linear models for microarray data approach. Cox proportional hazard regression analysis was applied to access the prognostic value of identified differentially methylated genes. The diagnostic value of the genes was evaluated through receiver operating characteristic. Pathway analyses were performed to illustrate biological functions of the identified genes. Compared to adjacent tissues, 77 genes with hypermethylated promoters and 2,412 genes with hypomethylated promoters were identified in HCC. The promoter hypomethylations of RNA5SP38, IL21, SDC4P, and MIR4439 were found to be associated with HCC patient survival $(P=0.035,0.040,0.004$, and 0.024 , respectively). Hypomethylated SDC4P was associated with a better prognosis (hazard ratio, $0.482 ; 95 \%$ confidence interval $[\mathrm{CI}],-0.147-1.110 ; P=0.007)$. The combination of the promoter hypomethylations with RNA5SP38, IL21, and SDC4P showed an area under receiver operating characteristic curves of 0.975 (95\% CI, 0.962-0.989; $P=4.811 \mathrm{E}-25)$. Several pathways, including olfactory transduction, cytokine-cytokine receptor interaction, natural killer cell-mediated cytotoxicity, as well as inflammation mediated by chemokine and cytokine signaling pathway, were annotated with the hypomethylated promoter genes. SDC4P promoter hypomethylation may be a potential prognosis biomarker. A panel of promoter methylations in RNA5SP38, IL21, and SDC4P was proven a novel approach to diagnosis HCC. The pathway analysis defined the extensive functional role of DNA hypomethylation in cancer.
\end{abstract}

Keywords: hepatocellular carcinoma, promoter methylation, prognosis, diagnosis

\section{Introduction}

Hepatocellular carcinoma (HCC) is a major health problem worldwide, which causes $\sim 600,000$ deaths every year. ${ }^{1}$ Liver transplantation, surgical resection, target therapy, and chemotherapy are currently available therapeutic strategies for HCC. ${ }^{2}$ However, the prognosis of HCC remains extremely dismal, with long-term 5-year survival rate ranging from $17 \%$ to $53 \% .{ }^{3,4} \mathrm{HCC}$ patients are commonly diagnosed at advanced stage, which may also contribute to the poor prognosis. ${ }^{5}$ Understanding the molecular mechanisms in HCC could help identify new therapy target and find effective diagnostic and prognostic biomarkers for HCC.

Accumulated evidences have demonstrated the vital roles of DNA methylation in many biological activities, especially in cancer initiation and progression. ${ }^{6-8}$ Regional hypermethylation and global hypomethylation are two common kinds of aberrant

\footnotetext{
Correspondence: Hong Cen

Department of Chemotherapy, Tumor Hospital of Guangxi Medical University, Hedi7I, Nanning 530000, Guangxi,

People's Republic of China

Tel +8607715390813

Fax +86 077I 5327596

Email hongcen_postdoctor@I63.com
} 
methylation in cancers. ${ }^{9}$ Some of the tumor-specific DNA methylations have been proposed to be potential diagnostic or prognostic biomarkers. ${ }^{10,11}$ Aberrant DNA methylations have been frequently found in HCC, which contributed to carcinogenesis by transcriptional silencing of tumor-suppressor genes (TSGs). ${ }^{12,13}$ Recent studies have attempted to find promising epigenetic aberrations to evaluate prognosis. ${ }^{14,15}$ Remarkably, DNA hypomethylation genes exhibit upregulated expression level in tumors and have effective impact upon tumor cell growth and metastasis. ${ }^{16}$ This group of genes is supposed to constitute targets of epigenetic therapy. However, most studies were conducted to investigate the association between a specific gene promoter methylation with cancer survival, rather than screening the association between genome-wide methylation and cancer survival. ${ }^{17,18}$ Few concordant gene methylation patterns were observed across the individual studies. Thus, we hypothesized that the study conducted in the manner of assessing global differential promoter methylation of genes and clinical features may provide in-depth results.

The Cancer Genome Atlas (TCGA) database contains a collection of genomic alterations, DNA methylation, RNA, proteomic expression, and clinicopathological data profiles, which could help explore the molecular characteristics of HCC comprehensively (Cancer Genome Atlas Research N 2013). With the aim to identify a methylation profile informative for HCC clinical features, we stringently conducted a stepwise study taking advantage of the data from TCGA project to 1) ascertain the differential promoter methylation expression profiles between HCC tumors and non-cancerous tissues, 2) identify the methylation associated with prognosis potential from the differential expression profiles, 3) find out methylation with reliable diagnosis potential, and 4) understand the biological pathways of the differential methylation profiles.

\section{Materials and methods}

\section{Patients and samples from TCGA}

All data for HCC patients were retrieved from TCGA data portal up to June 1, 2016. The data of the patients who have suffered from other malignancies or received neo-adjuvant therapy were not included. The full clinical information including sex, age, race, vital status, tumor grade, tumor pathologic stage, lymph node pathologic stage, metastasis stage, the American Joint Committee on Cancer (AJCC) pathologic stage, and methylation values (level 1 data, Illumina Infinium Human Methylation 450K) were then downloaded. Adjacent tissues were away from the tumor margin at least $2 \mathrm{~cm}$. Because the data were obtained from TCGA, further approval by an ethics committee was not required. This study meets the publication guidelines provided by TCGA (http://cancergenome.nih.gov/publicationguidelines).

\section{Illumina infinium human methylation $450 \mathrm{~K}$ analysis}

The differential methylated genes in the HCC tissues (Cohort T) and adjacent non-tumor tissues (Cohort N) were investigated. Our study calculated TCGA Illumina Human Methylation 450 array of HCC using RnBeads version 0.99.19 in the $\mathrm{R}$ software 3.1.2, where the methylation signal data were extracted and processed. In processing and filtering, a probe was filtered out when the last five bases in its target sequence overlapped with single-nucleotide polymorphism and removed $\mathrm{CpG}$ sites with $>10 \%$ missing values in all samples. Methylation measures with a detection $P$-value of $>0.01$ were removed. Both sites and samples were filtered using a greedy approach. In addition, $\mathrm{CpG}$ sites on the sex chromosomes were removed to avoid sex-specific methylation bias. Background subtraction with method "methylumi.noob" and normalization with method "bmiq" were performed, respectively. Surrogate variable analysis was performed to exclude covariates against the variable of tissue types (tumor and adjacent non-tumor tissues). The linear models for microarray data (LIMMA) package were used to identify differential methylated genes between HCC and adjacent tissues.

\section{Biological functions analysis}

To access main biological functions that were regulated by DNA methylation, we utilized differential methylated promoter genes for Kyoto Encyclopedia of Genes and Genomes (KEGG) pathway and Gene Ontology (GO) analyses based on the Database for Annotation, Visualization, and Integrated Discovery (DAVID). GO can organize genes into hierarchical categories and show the gene regulatory network, biological process, and molecular functions. ${ }^{19}$

\section{Statistical analysis}

The differences of clinical characteristics as mentioned above were evaluated using chi-square tests in GraphPad Prism 5.0 software. The univariate Cox proportional hazards regression analysis was firstly performed to elucidate the association between differential promoter methylated genes and overall survival (OS). Kaplan-Meier survival analysis and univariate/multivariate Cox proportional hazards regression analyses were sequentially carried out in IBM Statistical Package for the Social Sciences (SPSS) Statistics Version 
22.0. The predicted values from the logistic regression model were employed to construct receiver operating characteristic (ROC) curves and then to calculate the area under the ROC curves (AUC) through "pROC" package of R soft. $P$-values $<0.05$ were considered statistically significant.

\section{Results}

\section{Patient characteristics}

To explore the HCC whole-genome promoter DNA methylomes, we analyzed 327 HCC tumor tissues (Cohort T) and 46 adjacent non-tumor tissues (Cohort $\mathrm{N}$ ) using the TCGA Illumina Human Methylation 450 microarray. All 327 patients were pathologically diagnosed with HCC. After quality control and filtering, DNA methylation analysis on $323 \mathrm{HCC}$ and 45 adjacent tissues was carried out. These samples were collected between 1995 and 2013. The detailed clinical information is presented in Table 1. The mean age and standard deviation for all 323 patients were $59.040 \pm 12.885$ years. Among the 323 participants (Cohort T), a total of 45 patients with adjacent non-tumor tissues were included in Cohort N. As summarized in Table 1, no significant difference was observed in the distribution of sex $(P=0.239)$, tumor grade $(P=0.113)$, tumor pathologic stage $(P=0.523)$, node pathologic stage $(P=0.355)$, metastasis pathologic stage $(P=0.727)$, and AJCC pathologic stage $(P=0.052)$ between the two cohorts.

\section{Differential methylated genes within promoter between tumor and adjacent non-tumor tissues}

The study utilized LIMMA approach to identify the statistically different DNA methylation status between HCC and adjacent tissues, based on the remaining 445,428 probes. Promoter methylations of 2,489 genes were found to be significantly different with false discovery rate (FDR) $<0.05$ and $\mid$ delta beta $\mid>0.2$ bases on 323 cancer tissues and 45 control tissues. Compared to adjacent tissue, 77 genes were hypermethylated and 2,412 genes were hypomethylated within promoters in HCC, showing a surprisingly outstanding gene promoter hypomethylation in Table S1 and Figure 1. A differential methylated pattern was presented between HCC and adjacent tissues in Figure 2. Besides, the methylation profile in the 45 patients with both cancer tissues and adjacent non-tumor tissues was evaluated and is presented in Table S2, and the results were almost overlapped with those based on 323 cancer tissues and 45 control tissues. However, given the 323 cancer tissues had contained 45 cancer tissues, as well as to include more samples for survival and diagnostic
Table I Characteristics of patients with hepatocellular cell carcinoma from the TCGA data portal

\begin{tabular}{|c|c|c|c|}
\hline Category & Cohort N (n=45) & Cohort T $(n=323)$ & $P$-value \\
\hline \multicolumn{3}{|c|}{ Age (years) } & 0.072 \\
\hline$\leq 60$ & 16 & 161 & \\
\hline$>60$ & 29 & 162 & \\
\hline \multicolumn{3}{|l|}{ Sex } & 0.239 \\
\hline Female & 18 & 101 & \\
\hline Male & 27 & 222 & \\
\hline \multicolumn{3}{|l|}{ Race } & 0.000 \\
\hline Asian & 6 & 152 & \\
\hline African & 6 & 16 & \\
\hline White & 30 & 144 & \\
\hline NA & 3 & 11 & \\
\hline \multicolumn{3}{|c|}{ Vital status } & 0.000 \\
\hline Alive & 18 & 245 & \\
\hline Dead & 27 & 78 & \\
\hline \multicolumn{3}{|c|}{ Tumor grade } & 0.113 \\
\hline GI & 5 & 46 & \\
\hline G2 & 22 & 152 & \\
\hline G3 & 16 & 110 & \\
\hline G4 & 0 & 13 & \\
\hline NA & 2 & 2 & \\
\hline \multicolumn{3}{|c|}{ Tumor pathologic stage } & 0.523 \\
\hline TI & 21 & 159 & \\
\hline $\mathrm{T} 2$ & 16 & 88 & \\
\hline T3 & 5 & 62 & \\
\hline $\mathrm{T} 4$ & 3 & 13 & \\
\hline $\mathrm{TX}$ & 0 & I & \\
\hline \multicolumn{3}{|c|}{ Node pathologic stage } & 0.355 \\
\hline No & 30 & 234 & \\
\hline NI & I & 4 & \\
\hline$N X$ & 13 & 84 & \\
\hline NA & 1 & I & \\
\hline \multicolumn{3}{|c|}{ Metastasis pathologic stage } & 0.727 \\
\hline Mo & 32 & 245 & \\
\hline MI & I & 4 & \\
\hline MX & 12 & 74 & \\
\hline \multicolumn{3}{|c|}{ AJCC pathologic stage } & 0.052 \\
\hline Stage I & 20 & 151 & \\
\hline Stage II & 12 & 80 & \\
\hline Stage III & 5 & 70 & \\
\hline Stage IV & 1 & 6 & \\
\hline NA & 7 & 16 & \\
\hline
\end{tabular}

Note: Cohort $\mathrm{N}$ represents adjacent non-tumor tissues, while Cohort T represents hepatocellular carcinoma tissues.

Abbreviations: AJCC, American Joint Committee on Cancer; NA, not applicable; TCGA, The Cancer Genome Atlas.

analyses, only the results from 323 cancer tissues were chosen for further analyses.

\section{Genes with methylated promoter associated with HCC patient survival}

Follow-up data on survival were available for the 323 TCGA HCC patients, and 78 patients died by the end of follow-up. To identify the potential promoter methylations of genes with prognostic characteristics, a total of 864 differential 


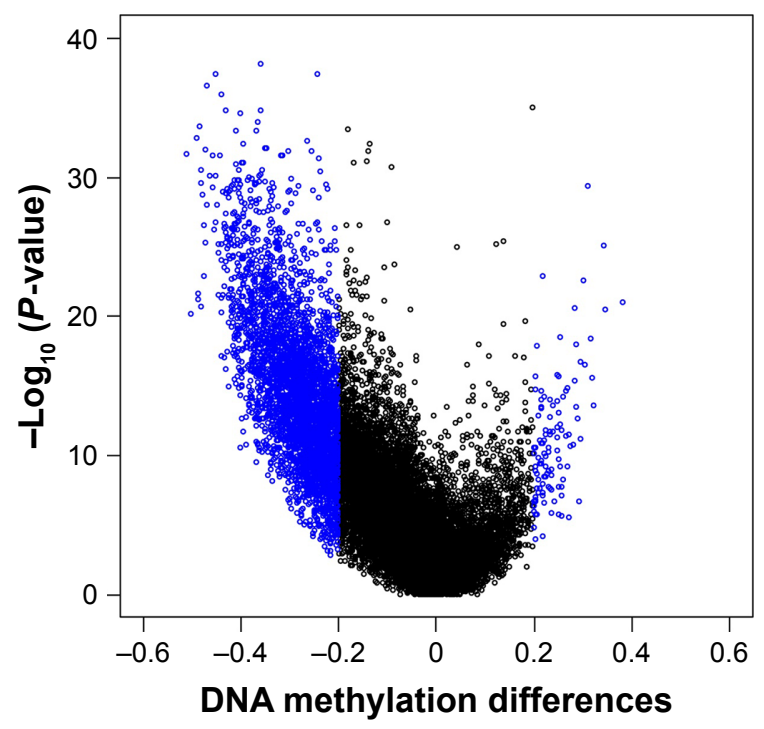

Figure I Volcano plot showing the distribution of genes from the promoter-level test assessed by methylation differences and adjusted $P$-values.

Note: All of gene promoters are with FDR $<0.05$, gene promoters in blue are differentially methylated $>0.2$ or $<-0.2$.

Abbreviation: FDR, false discovery rate.

methylated genes within promoter (FDR $<0.05$, delta beta| $>0.3$ ) were subjected to univariate Cox proportional hazard regression analysis. Then four promoter hypomethylations of genes: RNA 5S ribosomal pseudogene 38 (RNA5SP38), interleukin-21 (IL21), syndecan 4 pseudogene

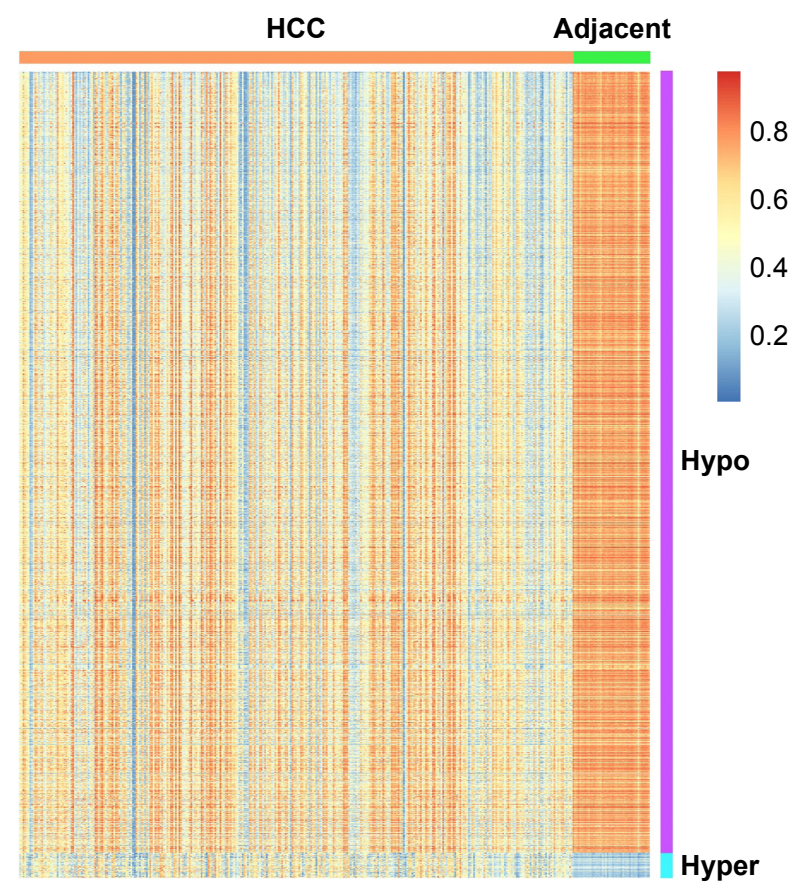

Figure 2 Heatmap of differentially methylated genes within promoters between $\mathrm{HCC}$ cancer tissues and adjacent tissues.

Notes: Hierarchical clustering by $323 \mathrm{HCC}$ cancer tissues (orange color bar) and 45 adjacent tissues (green color bar) with 2,489 significant genes within promoters (FDR $<0.05$, |delta beta| $>0.2$ ); red/blue gradient represents beta values of the methylations.

Abbreviations: FDR, false discovery rate; HCC, hepatocellular carcinoma.
(SDC4P), and microRNA 4439 (MIR4439) were found to be associated with HCC patient survival $(P=0.035,0.040,0.004$, and 0.024 , respectively). Multivariate Cox proportional hazard regression analysis was further performed to determine whether the four gene promoter methylations could be independent prognostic factors (Table 2). Under the control of potential confounding factors, including sex, node stage, and metastasis stage, we concluded that RNA5SP38, IL21, and SDC4P promoter methylations would be individually independent prognostic variables $(P=0.008,<0.001$, and 0.035 , respectively), but not MIR4439 promoter methylation $(P=0.419)$.

The median levels of RNA5SP38, IL21, and SDC4P promoter methylations were cutoff, respectively. KaplanMeier survival curves stratified by high and low levels of RNA5SP38, IL21, and SDC4P promoter methylation are shown in Figure 3. Log-rank test showed that lower methylated levels of SDC4P promoter have a significantly favorable prognosis for $\mathrm{HCC}$ patients (hazard ratio $=0.482$, 95\% confidence interval $[\mathrm{CI}]=-0.147-1.110, P=0.007)$, while not in RNA5SP38 $(P=0.108)$ and IL21 $(P=0.127)$ promoter methylations. HCC patients with low methylated levels of SDC4P promoter survived longer than those with high methylated levels of SDC4P promoter.

\section{Diagnostic value of promoter methylations of RNA5SP38, IL2I, and SDC4P}

ROC analysis was applied to calculate the significance of the promoter methylations to RNA5SP38, IL21, and SDC4P as potential biomarkers to differentiate $\mathrm{HCC}$ tissues from adjacent tissues (Figure 4). The promoter methylation of SDC4P (AUC $=0.935,95 \% \mathrm{CI}=0.909-0.961, P=3.211 \mathrm{E}-21)$ demonstrated a higher diagnostic capability than promoter methylations of RNA5SP38 (AUC $=0.923,95 \%$ CI $=0.895-0.951$, $P=3.585 \mathrm{E}-20$ ) and promoter methylation of IL21 (AUC $=0.907$, $95 \% \mathrm{CI}=0.877-0.937, P=9.248 \mathrm{E}-19)$. Subsequently, the logistic regression approach was applied for the combination of these three genes with promoter methylations, and the ROC curve revealed a much better diagnostic accuracy than when they were calculated individually. The optimal cutoff value was set at a maximal sum of sensitivity and specificity. The result showed an AUC of 0.975 (95\% CI $=0.962-0.989$, $P=4.811 \mathrm{E}-25)$, and the diagnosis sensitivity and specificity of the combination of these three promoter methylations were found to be $91.3 \%$ and $100.0 \%$, respectively.

\section{KEGG pathway and GO analysis}

To avoid bias from promoter hypermethylation genes, only the substantial promoter hypomethylation genes were 
Table 2 Univariate and multivariate analysis of prognostic variables for cancer-specific survival in patients with hepatocellular cell carcinoma

\begin{tabular}{|c|c|c|c|c|c|}
\hline \multirow[t]{2}{*}{ Variables } & \multirow{2}{*}{$\begin{array}{l}\text { No. of } \\
\text { patients }\end{array}$} & \multicolumn{2}{|c|}{ Univariate analysis } & \multicolumn{2}{|c|}{ Multivariate analysis } \\
\hline & & $P$-value & Hazard ratios $(95 \% \mathrm{Cl})$ & $P$-value & Hazard ratios $(95 \% \mathrm{Cl})$ \\
\hline \multicolumn{6}{|l|}{ Age (years) } \\
\hline$>60$ & 162 & 0.063 & & - & - \\
\hline$\leq 60$ & 161 & & $1.558(0.976-2.486)$ & & \\
\hline \multicolumn{6}{|l|}{ Sex } \\
\hline Female & 101 & 0.040 & & & \\
\hline Male & 222 & & $\mathrm{I} .605(\mathrm{I} .02 \mathrm{I}-2.52 \mathrm{I})$ & 0.344 & $0.781(0.468-1.304)$ \\
\hline \multicolumn{6}{|l|}{ Tumor stage } \\
\hline $\mathrm{pTI}+\mathrm{pT} 2$ & 247 & 0.205 & & - & - \\
\hline $\mathrm{pT} 3+\mathrm{p} T 4+\mathrm{p} T \mathrm{X}$ & 76 & & $\mathrm{I} .414(0.827-2.417)$ & & \\
\hline \multicolumn{6}{|l|}{ Node stage } \\
\hline pNO & 238 & 0.005 & & & \\
\hline $\mathrm{pNX}$ & 84 & & $2.020(1.238-3.297)$ & 0.311 & $1.454(0.704-3.000)$ \\
\hline \multicolumn{6}{|l|}{ Metastasis stage } \\
\hline $\mathrm{pMO}+\mathrm{pMI}$ & 249 & 0.002 & & & \\
\hline pMX & 74 & & $2.186(1.348-3.546)$ & 0.264 & I.533 (0.724-3.245) \\
\hline \multicolumn{6}{|l|}{ Fuhrman grade } \\
\hline $\mathrm{Gl}+\mathrm{G} 2$ & 198 & 0.086 & & - & - \\
\hline $\mathrm{G} 3+\mathrm{G} 4$ & 123 & & $1.495(0.944-2.366)$ & & \\
\hline \multicolumn{6}{|l|}{ Pathological stage } \\
\hline$I+I I$ & 234 & 0.502 & & - & - \\
\hline III + IV & 73 & & $\mathrm{I} .232(0.67 \mathrm{I}-2.26 \mathrm{I})$ & & \\
\hline RNA5SP38 promoter & 323 & 0.035 & $0.294(0.094-0.917)$ & 0.008 & $0.14 \mid(0.033-0.605)$ \\
\hline IL2I promoter & 323 & 0.040 & $4.653(1.073-20.173)$ & $<0.00$ I & $32.706(5.642-189.582)$ \\
\hline SDC4P promoter & 323 & 0.004 & $0.157(0.045-0.549)$ & 0.035 & $0.163(0.030-0.883)$ \\
\hline MIR4439 promoter & 323 & 0.024 & $0.256(0.079-0.836)$ & 0.419 & $0.532(0.1|5-2.46|)$ \\
\hline
\end{tabular}

Abbreviations: $\mathrm{Cl}$, confidence of interval; IL2I, interleukin-2I; MIR4439, microRNA 4439; RNA5SP38, RNA 5 S ribosomal pseudogene 38; SDC4P, syndecan 4 pseudogene.

subjected to KEGG and GO analysis. To identify pathways and biological functions that enriched by genes with differential hypomethylation promoter, we analyzed GO and KEGG pathways through the 2,412 genes and displayed the GO results in Table S3. In the KEGG pathway, the genes showed dominant enrichments in the pathways of olfactory transduction $(P=5.740 \mathrm{E}-188)$, cytokine-cytokine receptor interaction $(P=1.962 \mathrm{E}-02)$, natural killer cell-mediated cytotoxicity $(P=8.702 \mathrm{E}-03)$, and inflammation mediated by chemokine and cytokine signaling pathway $(P=4.300 \mathrm{E}-04)$, which are shown in Table 3. Besides, GO analysis revealed the biological processes, cellular components, and molecular functions of differentially hypomethylated genes within promoter.
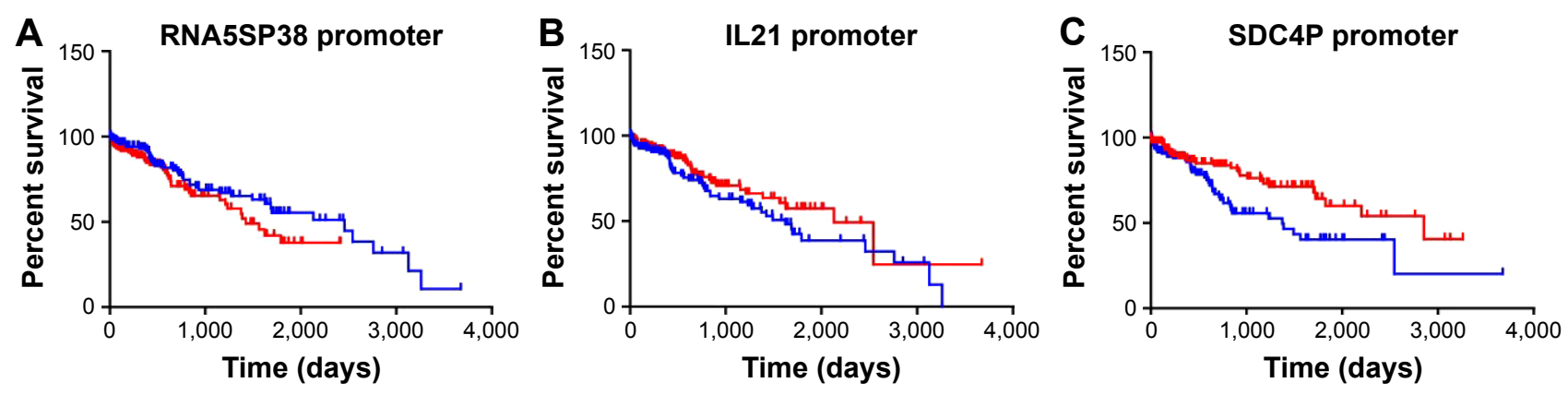

$\perp$ Low methylation + High methylation

Figure 3 Kaplan-Meier plot of overall survival.

Notes: (A) Overall survival for HCC with high and low methylation levels of the RNA5SP38 promoter; (B) overall survival for HCC with high and low methylation levels of the IL2I promoter; (C) overall survival for HCC with high and low methylation levels of the SDC4P promoter.

Abbreviations: HCC, hepatocellular carcinoma; IL2I, interleukin-2I; RNA5SP38, RNA 5S ribosomal pseudogene 38; SDC4P, syndecan 4 pseudogene. 
A

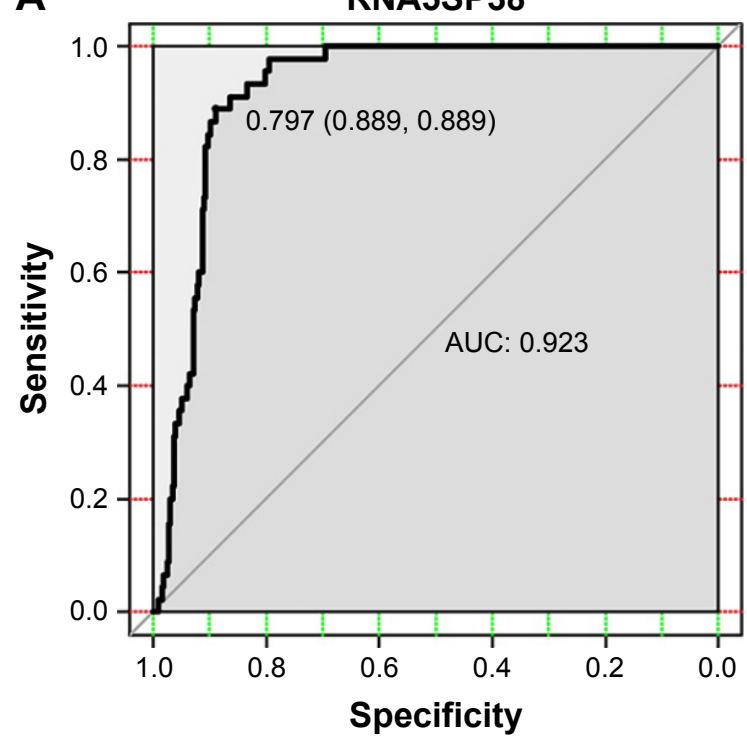

C

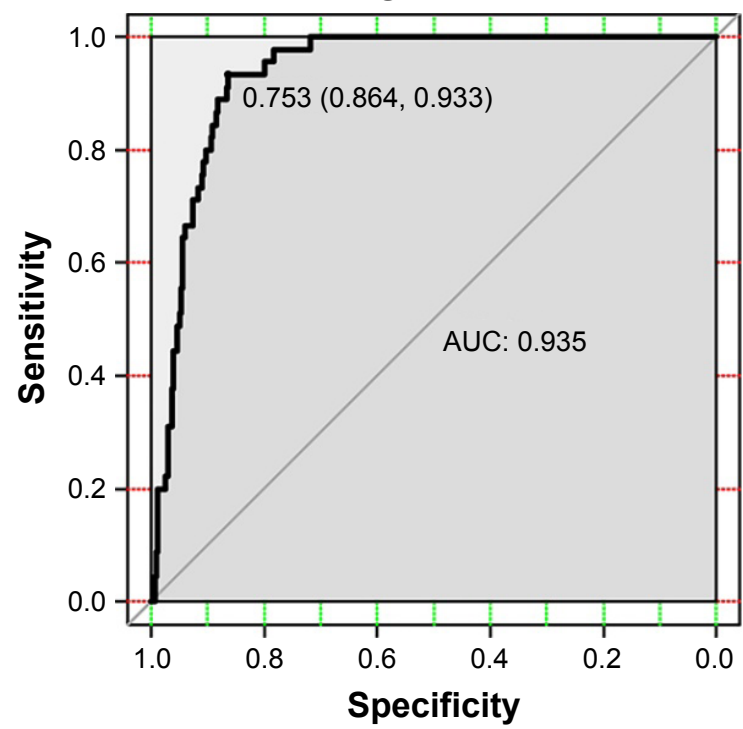

B

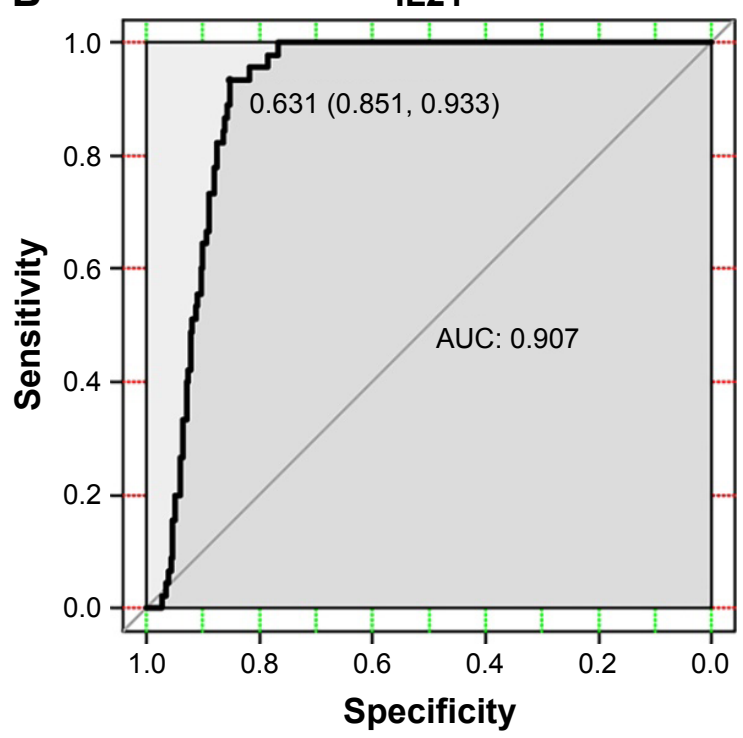

D

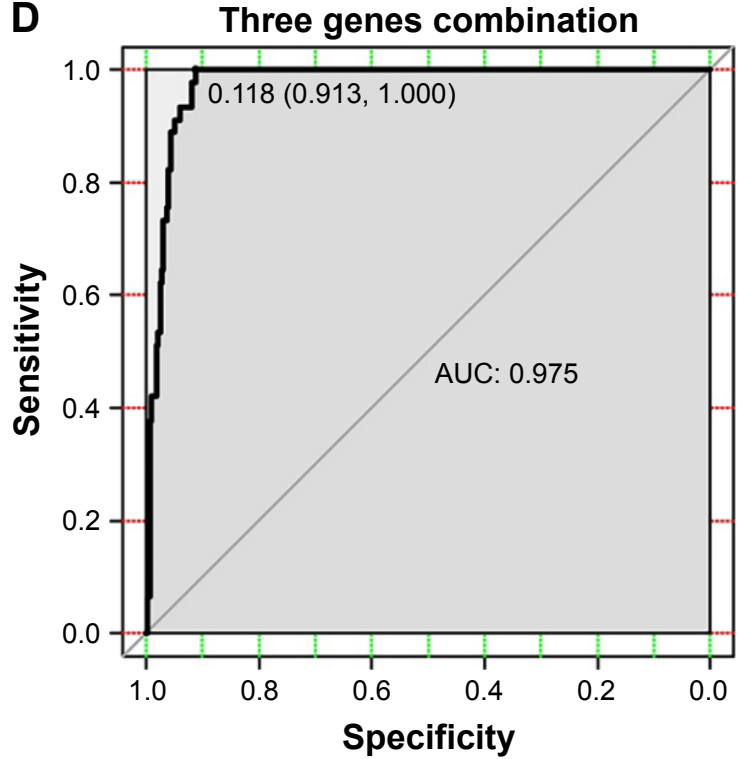

Figure 4 The areas under the ROC curves for the three gene promoter methylations and their combination that differentiate between HCCs and adjacent tissues. Notes: (A) The ROC curve for the RNA5SP38 promoter. (B) The ROC curve for the IL2I promoter. (C) The ROC curve for the SDC4P promoter. (D) The ROC curve for the combination of the three gene promoter methylations.

Abbreviations: AUC, area under the curve; HCC, hepatocellular carcinoma; IL2I, interleukin-2I; RNA5SP38, RNA 5S ribosomal pseudogene 38; ROC, receiver operating characteristic; SDC4P, syndecan 4 pseudogene.

Table 3 KEGG and PANTHER pathways enriched in differentially methylated genes within promoters

\begin{tabular}{llll}
\hline Category & Term & Count genes & P-value \\
\hline KEGG & hsa04740: olfactory transduction & 254 & $5.740 E-188$ \\
KEGG & hsa05332: graft-versus-host disease & 12 & $8.876 \mathrm{E}-04$ \\
KEGG & hsa05320: autoimmune thyroid disease & 14 & $8.926 \mathrm{E}-04$ \\
KEGG & hsa04650: natural killer cell-mediated cytotoxicity & 23 & $8.702 \mathrm{E}-03$ \\
KEGG & hsa04060: cytokine-cytokine receptor interaction & 37 & $1.962 \mathrm{E}-02$ \\
KEGG & hsa04640: hematopoietic cell lineage & 16 & $1.764 \mathrm{E}-02$ \\
KEGG & hsa046I2: antigen processing and presentation & 15 & $2.800 \mathrm{E}-02$ \\
KEGG & hsa054I6: viral myocarditis & 13 & $3.959 \mathrm{E}-02$ \\
PANTHER & P0003I: inflammation mediated by chemokine and cytokine signaling pathway & 29 & $4.300 \mathrm{E}-04$ \\
\hline
\end{tabular}

Abbreviation: KEGG, Kyoto Encyclopedia of Genes and Genomes. 


\section{Discussion}

TCGA dataset provides a powerful approach to investigate the association between epigenetic alterations and clinical outcomes, paving way to elucidate new diagnostic and prognostic markers..$^{20}$ Herein, we gained 2,412 hypomethylated and 77 hypermethylated promoter genes between tumor and adjacent non-tumor tissues. Stefanska et al reported that nearly 2,000 genes within promoter hypomethylation were found in another HCC epigenetic study. ${ }^{21}$ These evidences suggested that the broad scope of DNA hypomethylation influenced HCC profoundly and potential biomarkers might be detected from hypomethylated genes. Some hypermethylated promoter genes identified in the current study have been reported in recently published studies, such as TBX15, RASSF10, AKR1B1, CDKL2, and ZNF154. ${ }^{10,13}$ Consistent with a previous study, S100A8 was presented a lower methylation level in HCC tissues compared to adjacent tissues. ${ }^{22}$ Hypermethylation of TSGs was considered as one of the hallmarks in cancer initiation and progression, while the effects of these alterations were mostly proven to depress TSGs expression in several kinds of malignancies. ${ }^{23,24}$ On the contrary, promoter hypomethylation in genes was observed related to the activation of oncogenes and pro-metastatic genes in HCC. ${ }^{21,25}$

This study has found four promoter hypomethylations of RNA5SP38, IL21, SDC4P, and MIR4439 that may be associated with HCC patient survival. RNA5SP38, IL21, and SDC4P promoter methylations would be individually independent prognostic variables. Noteworthy, RNA5SP38 and SDC4P were pseudogenes and found to be associated with HCC survival for the first time. A great number of pseudogenes have been discovered to play an important role in cancer through relating activity to parental genes or functioning independently. ${ }^{26}$ It was becoming clear that certain pseudogenes were also transcribed and maintained functions. ${ }^{27}$ The association between pseudogene methylation and cancer progression should be considered exploratory. ${ }^{28}$ In a pan-cancer analysis of pseudogene, it was reported that the pseudogene expression subtypes not only significantly related to patient survival, but also enabled to stratify patients in combination with clinical variables in kidney cancer. ${ }^{29}$ $\mathrm{Yu}$ et al have illustrated that pseudogene methylation could downregulate the corresponding gene expression, which might contribute to clear-cell renal cell carcinoma progression. ${ }^{30}$ Another noteworthy gene was IL21, which was discovered in 2000 and has been known as an important regulator of immune responses. It harbored pleiotropic functions dominantly at modulating the activity of $\mathrm{T}$ cells,
B cells, and natural killer cells. ${ }^{31}$ IL21 contributed to antitumor effects by direct and immune-mediated cytotoxicity. ${ }^{32} \mathrm{Com}-$ bined with other factors, IL21 had potent to predict the outcome of ovarian cancer patients..$^{33}$ Our study has suggested the potential prognostic role of promoter hypomethylation of RNA5SP38, IL21, and SDC4P in HCC patients for the first time. Furthermore, the study revealed that hypomethylation of SDC4P was associated with a better outcome, suggesting that SDC4P might be a potential TSG for HCC.

Our study demonstrated the significant diagnostic value of the combination of RNA5SP38, IL21, and SDC4P. It was not surprising that a panel of genes with promoter hypomethylation displayed better diagnosis accuracy than an individual methylated alteration. Aberrant methylations occurred far more frequently than genetic mutations in cancers. ${ }^{34}$ Thus, identification of specific DNA methylation signatures gained robust potential to generate diagnostic markers for detection of HCC..$^{35}$ It was notable that our study provided this diagnostic finding based on the TCGA data, most of which were of early stage HCC patients (stage I + stage II, accounting for $71.5 \%$ HCC patients). To some extent, this result may indicate a novel diagnostic approach for the early HCC detection. Future clinically verified experiments are in great demand.

The signaling pathway analysis showed that cytokinecytokine receptor interaction, natural killer cell-mediated cytotoxicity, and inflammation mediated by chemokine and cytokine signaling pathway were correlated with HCC. GO annotation yielded multiple signaling pathways in the biological process, cellular component, and molecular function. They linked to DNA replication, cell cycle, immune response, and defense response. All these data together indicated that multiple pathways, which were influenced by hypomethylation phenomenon, were involved in comprehensive cell developmental processes coordinately. The immune system was essential in body defense system, and the dysfunction of immune system might be correlated with cancer initiation. ${ }^{36}$ Interestingly, a large number of identified genes were enriched in olfactory transduction pathway in KEGG analysis. Likewise, similar pathways (olfactory receptor activity, sensory perception of chemical stimulus, and sensory perception) were obtained by GO analysis. Previous reports have shown widespread ectopic olfactory receptor expression in non-olfactory tissues..$^{37,38}$ A study reported that olfactory receptor activation increased intracellular $\mathrm{Ca}^{2+}$ and reduced cell proliferation in Huh7 HCC cell line..$^{39}$ Olfactory receptor activation was also observed to inhibit human leukemia cell proliferation, evoke apoptosis, 
and regulate differentiation. ${ }^{40}$ Although the association between the olfactory transduction and HCC remained unclear, the current evidences suggested the potential antitumor behavior of olfactory transduction.

With regard to limitations of this study, we were unable to conduct stratified analysis based on hepatitis B virus (HBV) infection status using the available TCGA data. HBV infection constituted the major etiology in Asian HCC patients, whereas fatty liver disease and high alcohol consumption were dominant in the white race. ${ }^{41,42}$ Our study might have missed some significant differential methylated promoter genes.

\section{Conclusion}

In summary, our data suggested that SDC4P promoter hypomethylation may be a potential prognostic biomarker. A panel of promoter methylation in RNA5SP38, IL21, and SDC4P was proven a novel approach to diagnosis HCC. The pathway analysis defined the wide functional role of DNA hypomethylation in cancer. A future study to validate the potential prognostic and diagnostic biomarkers in $\mathrm{HCC}$ patients is necessary.

\section{Acknowledgment}

The authors acknowledge the great contributions of The Cancer Genome Atlas database.

\section{Disclosure}

The authors report no conflicts of interest in this work.

\section{References}

1. Torre LA, Bray F, Siegel RL, Ferlay J, Lortet-Tieulent J, Jemal A. Global cancer statistics, 2012. CA Cancer J Clin. 2015;65(2):87-108.

2. Forner A, Llovet JM, Bruix J. Hepatocellular carcinoma. Lancet. 2012; 397(9822):1245-1255.

3. Byam J, Renz J, Millis JM. Liver transplantation for hepatocellular carcinoma. Hepatobiliary Surg Nutr. 2013;2(1):22-30.

4. Singhal A, Jayaraman M, Dhanasekaran DN, Kohli V. Molecular and serum markers in hepatocellular carcinoma: predictive tools for prognosis and recurrence. Crit Rev Oncol Hematol. 2012;82(2):116-140.

5. Cheng AL, Kang YK, Chen Z, et al. Efficacy and safety of sorafenib in patients in the Asia-Pacific region with advanced hepatocellular carcinoma: a phase III randomised, double-blind, placebo-controlled trial. Lancet Oncol. 2009;10(1):25-34.

6. Kong HK, Park SJ, Kim YS, et al. Epigenetic activation of LY6K predicts the presence of metastasis and poor prognosis in breast carcinoma. Oncotarget. 2016;7(34):55677-55689.

7. Paska AV, Hudler P. Aberrant methylation patterns in cancer: a clinical view. Biochem Med (Zagreb). 2015;25(2):161-176.

8. Wu WR, Sun H, Zhang R, et al. Methylation-associated silencing of miR-200b facilitates human hepatocellular carcinoma progression by directly targeting BMI1. Oncotarget. 2016;7(14):18684-18693.

9. Ruan P, Shen J, Santella RM, Zhou S, Wang S. NEpiC: a network-assisted algorithm for epigenetic studies using mean and variance combined signals. Nucleic Acids Res. 2016;44(16):e134.
10. Wang F, Feng Y, Li P, et al. RASSF10 is an epigenetically inactivated tumor suppressor and independent prognostic factor in hepatocellular carcinoma. Oncotarget. 2016;7(4):4279-4297.

11. Zhu X, Shan L, Wang F, et al. Hypermethylation of BRCA1 gene: implication for prognostic biomarker and therapeutic target in sporadic primary triple-negative breast cancer. Breast Cancer Res Treat. 2015; 150(3):479-486.

12. Li QF, Li QY, Gao AR, Shi QF. Correlation between promoter methylation in the GSTP1 gene and hepatocellular carcinoma development: a meta-analysis. Genet Mol Res. 2015;14(2):6762-6772.

13. Yamada N, Yasui K, Dohi O, et al. Genome-wide DNA methylation analysis in hepatocellular carcinoma. Oncol Rep. 2016;35(4):2228-2236.

14. Zhang X, You Q, Zhang X, Chen X. SOCS3 methylation predicts a poor prognosis in HBV infection-related hepatocellular carcinoma. Int J Mol Sci. 2015;16(9):22662-22675.

15. Lv Z, Zou H, Peng K, et al. The suppressive role and aberrent promoter methylation of BTG3 in the progression of hepatocellular carcinoma. PLoS One. 2013;8(10): 77437.

16. Stefanska B, Cheishvili D, Suderman M, et al. Genome-wide study of hypomethylated and induced genes in patients with liver cancer unravels novel anticancer targets. Clin Cancer Res. 2014;20(12):3118-3132.

17. Qiu X, Huang Y, Zhou Y, Zheng F. Aberrant methylation of TRIM58 in hepatocellular carcinoma and its potential clinical implication. Oncol Rep. 2016;36(2):811-818.

18. Sun FK, Sun Q, Fan YC, et al. Methylation of tissue factor pathway inhibitor 2 as a prognostic biomarker for hepatocellular carcinoma after hepatectomy. J Gastroenterol Hepatol. 2016;31(2):484-492.

19. Ashburner M, Ball CA, Blake JA, et al. Gene ontology: tool for the unification of biology. The gene ontology consortium. Nat Genet. 2000;25(1): 25-29.

20. Barbano R, Palumbo O, Pasculli B, et al. A miRNA signature for defining aggressive phenotype and prognosis in gliomas. PLoS One. 2014; 9(10):e108950

21. Stefanska B, Huang J, Bhattacharyya B, et al. Definition of the landscape of promoter DNA hypomethylation in liver cancer. Cancer Res. 2011; 71(17):5891-5903.

22. Liu K, Zhang Y, Zhang C, et al. Methylation of S100A8 is a promising diagnosis and prognostic marker in hepatocellular carcinoma. Oncotarget. 2016;7(35):56798-56810.

23. Li W, Li X, Wang W, et al. NOR1 is an HSF1- and NRF1-regulated putative tumor suppressor inactivated by promoter hypermethylation in nasopharyngeal carcinoma. Carcinogenesis. 2011;32(9):1305-1314.

24. Zhang C, Peng Y, Yang F, Qin R, Liu W, Zhang C. PCDH8 is frequently inactivated by promoter hypermethylation in liver cancer: diagnostic and clinical significance. J Cancer. 2016;7(4):446-452.

25. Stefanska B, Bouzelmat A, Huang J, et al. Discovery and validation of DNA hypomethylation biomarkers for liver cancer using HRM-specific probes. PLoS One. 2013;8(8):e68439.

26. Grander D, Johnsson P. Pseudogene-expressed RNAs: emerging roles in gene regulation and disease. Curr Top Microbiol Immuol. 2016;394: $111-126$.

27. Kalyana-Sundaram S, Kumar-Sinha C, Shankar S, et al. Expressed pseudogenes in the transcriptional landscape of human cancers. Cell. 2012;149(7):1622-1634.

28. Byun DS, Cho K, Ryu BK, et al. Frequent monoallelic deletion of PTEN and its reciprocal association with PIK3CA amplification in gastric carcinoma. Int J Cancer. 2003;104(3):318-327.

29. Han L, Yuan Y, Zheng S, et al. The pan-cancer analysis of pseudogene expression reveals biologically and clinically relevant tumour subtypes. Nat Commun. 2014;5:3963.

30. Yu G, Yao W, Gumireddy K, et al. Pseudogene PTENP1 functions as a competing endogenous RNA to suppress clear-cell renal cell carcinoma progression. Mol Cancer Ther. 2014;13(12):3086-3097.

31. Leonard WJ, Spolski R. Interleukin-21: a modulator of lymphoid proliferation, apoptosis and differentiation. Nat Rev Immunol. 2005;5(9): 688-698. 
32. Bhatt S, Matthews J, Parvin S, et al. Direct and immune-mediated cytotoxicity of interleukin-21 contributes to antitumor effects in mantle cell lymphoma. Blood. 2015;126(13):1555-1564.

33. Chen YL, Chou CY, Chang MC, et al. IL17a and IL21 combined with surgical status predict the outcome of ovarian cancer patients. Endocr Relat Cancer. 2015;22(5):703-711.

34. Kulis M, Esteller M. DNA methylation and cancer. Adv Genet. 2010; 70:27-56.

35. Harada K, Baba Y, Ishimoto T, et al. LINE-1 methylation level and patient prognosis in a database of 208 hepatocellular carcinomas. Ann Surg Oncol. 2015;22(4):1280-1287.

36. Liu P, Jiang W, Ren H, Zhang H, Hao J. Exploring the molecular mechanism and biomakers of liver cancer based on gene expression microarray. Pathol Oncol Res. 2015;21(4):1077-1083.

37. Wiese H, Gelis L, Wiese S, et al. Quantitative phosphoproteomics reveals the protein tyrosine kinase Pyk 2 as a central effector of olfactory receptor signaling in prostate cancer cells. Biochim Biophys Acta. 2015; 1854(6):632-640.
38. Feldmesser E, Olender T, Khen M, et al. Widespread ectopic expression of olfactory receptor genes. BMC Genomics. 2006;7:121.

39. Massberg D, Simon A, Haussinger D, Yanai I, Ophir R, Lancet D. Monoterpene (-)-citronellal affects hepatocarcinoma cell signaling via an olfactory receptor. Arch Biochem Biophy. 2015;566:100-109.

40. Manteniotis S, Wojcik S, Brauhoff P, et al. Functional characterization of the ectopically expressed olfactory receptor 2AT4 in human myelogenous leukemia. Cell Death Discov. 2016;2:15070.

41. Kim BK, Han KH, Ahn SH. Prevention of hepatocellular carcinoma in patients with chronic hepatitis B virus infection. Oncology. 2011; (81 Suppl 1):41-49.

42. McGlynn KA, London WT. The global epidemiology of hepatocellular carcinoma: present and future. Clin Liver Dis. 2011;15(2):223-243.
OncoTargets and Therapy

\section{Publish your work in this journal}

OncoTargets and Therapy is an international, peer-reviewed, open access journal focusing on the pathological basis of all cancers, potential targets for therapy and treatment protocols employed to improve the management of cancer patients. The journal also focuses on the impact of management programs and new therapeutic agents and protocols on

\section{Dovepress}

patient perspectives such as quality of life, adherence and satisfaction. The manuscript management system is completely online and includes a very quick and fair peer-review system, which is all easy to use. Visit http://www.dovepress.com/testimonials.php to read real quotes from published authors. 